\title{
Valproate-Induced Liver Injury: Modulation by the Omega-3 Fatty Acid DHA Proposes a Novel Anticonvulsant Regimen
}

\author{
Marwa A. Abdel-Dayem • Ahmed A. Elmarakby • \\ Azza A. Abdel-Aziz $\cdot$ Chelsey Pye $\cdot$ Shehta A. Said • \\ Abdalla M. El-Mowafy
}

Published online: 15 April 2014

(C) The Author(s) 2014. This article is published with open access at Springerlink.com

\begin{abstract}
Background The polyunsaturated, $\omega-3$ fatty acid, docosahexaenoic acid (DHA), claims diverse cytoprotective potentials, although via largely undefined triggers. Thus, we currently first tested the ability of DHA to ameliorate valproate (VPA)-evoked hepatotoxicity, to modulate its anticonvulsant effects, then sought the cellular and molecular basis of such actions. Lastly, we also verified whether DHA may kinetically alter plasma levels/clearance rate of VPA.

Methods and Results VPA $(500 \mathrm{mg} / \mathrm{kg}$ orally for 14 days in rats) evoked prominent hepatotoxicity that appeared as a marked rise (2- to 4-fold) in serum hepatic enzymes $(\gamma$-glutamyl transferase $[\gamma-\mathrm{GT}]$, alanine aminotransferase [ALT], and alkaline phosphatase [ALP]), increased hepatic
\end{abstract}

S. A. Said: Deceased.

M. A. Abdel-Dayem · S. A. Said

Department of Pharmacology, Faculty of Pharmacy (FOP), Mansoura University, 35512 Mansoura, Egypt

A. A. Elmarakby · C. Pye

Department of Oral Biology/Pharmacology, Georgia Regents

University, Augusta, GA 30912, USA

A. A. Abdel-Aziz

Department of Pathology, Faculty of Medicine, Mansoura University, 35512 Mansoura, Egypt

A. M. El-Mowafy

Department of Clinical Biochemistry, Faculty of Pharmacy (FOP), Mansoura University, 35512 Mansoura, Egypt

A. M. El-Mowafy ( $\square)$

Department of Pharmacology, Faculty of Pharmacy, Future

University (FUE), 12311 New Cairo, Egypt

e-mail: aelmowafy@yahoo.com lipid peroxide (LPO) and tumor necrosis factor-alpha $(\mathrm{TNF} \alpha)$ levels, as well as myeloperoxidase (MPO) activity (3- to 5-fold), lowering of serum albumin (40\%), and depletion of liver reduced-glutathione (GSH, $35 \%$ ). Likewise, histopathologic examination revealed hepatocellular degeneration, replacement by inflammatory cells, focal pericentral necrosis, and micro/macrovesicular steatosis. Concurrent treatment with DHA $(250 \mathrm{mg} / \mathrm{kg})$ markedly blunted the elevated levels of liver enzymes, lipid peroxides, $\mathrm{TNF} \alpha$, and MPO activity, while raising serum albumin and hepatic GSH levels. DHA also alleviated most of the cytologic insults linked to VPA. Besides, in a pentylenetetrazole (PTZ) mouse convulsion model, DHA $(250 \mathrm{mg} / \mathrm{kg})$ markedly increased the latency in convulsion evoked by VPA, beyond their individual responses. Lastly, pharmacokinetic studies revealed that joint DHA administration did not alter serum VPA concentrations.

Conclusions DHA substantially ameliorated liver injury induced by VPA, while also markedly boosted its pharmacologic effects. DHA manipulated definite cellular machinery to curb liver oxidative stress and inflammation, without affecting VPA plasma levels. Collectively, these protective and synergy profiles for DHA propose a superior VPA-drug combination regimen.

\section{Introduction}

An increasing emphasis is being placed on the capacity of dietary supplements to modulate host response to disease, injury, infection, and adverse drug reactions [1-3]. It is estimated that drug-induced adverse reactions account for at least 5-6 \% of hospital admissions [4]. Valproate (VPA) is a widely prescribed fatty acid (FA) that has served as a mainstay in the management of epileptic seizures, bipolar 
and schizoaffective disorders, social phobias, and neuropathic pain [5]. Despite its clinical benefits, VPA has also been a hallmark representative of drug-induced adverse reactions. In particular, patients receiving VPA chronically may well develop hemorrhagic pancreatitis, bone marrow suppression and, more frequently, hepatic injury [6]. Thus, in up to $44 \%$ of patients, chronic dosing with VPA elevates serum liver enzymes and lipid peroxidation during the first months of therapy. Another typical clinical finding of VPA intoxication was the development of fatty liver as microvesicular steatosis in $80 \%$ of patients [7]. Moreover, animal models of VPA toxicity clearly show disruption of FA metabolism, along with accumulation of liver lipid content within 2-4 hours after VPA administration [8]. Histopathologic and biochemical studies also revealed that VPA evokes hepatic necrosis, apoptosis, and oxidative stress $[9,10]$. However, VPA toxicity that can lead to death has also been reported. The basis of such paradoxical subacute and idiosyncratic VPA toxicity has remained largely enigmatic [11].

At the molecular level, multiple lines of evidence suggest that hepatic accumulation of 4-en-VPA and its $\beta$ oxidation products triggers a cascade of reactions that culminates in hepatic injury. Some such reactions involve lipid peroxidation and glutathione (GSH) depletion [12, 13]. Conceivably, therefore, a big need arises to seek avenues that could either alleviate VPA-induced hepatic injury or reduce its dose down to a safer level, thus possibly improving its overall therapeutic index. Thus far, diverse concepts have been adopted, which focused merely on lessening oxidative stress or disrupted mitochondrial fattyacyl $\beta$-oxidation $[14,15]$. Conversely, no attempts have been made to boost the pharmacologic efficacy of VPA so as to reduce its toxicity, while also augmenting its therapeutic efficacy. Docosahexaenoic acid (DHA) is a coldwater-fish-oil-derived omega-3 FA that has demonstrated numerous health benefits against malignant, inflammatory, proliferative, and vascular diseases [16]. Furthermore, we recently demonstrated that DHA can reverse a vicious, fatal, cisplatin-induced nephrotoxicity in rats by ablating oxidative stress and suppressing cytokine-mediated inflammation [17]. As far as central effects are concerned; DHA was effectively used to treat neuronal hyperexcitability models in animals and some neurological disorders in humans $[18,19]$. Therefore, we currently envisaged that such responses, along with established hypolipidemic effects elicited mostly at the liver level [20], could make DHA supplementation a superb candidate to blunt toxicity and confer therapeutic synergy with VPA.

Accordingly, this study was marshaled to investigate whether, and how, DHA may abate VPA-induced liver toxicity. To accomplish this, we monitored levels of hepatocellular oxidative stress, inflammatory cytokines, and markers for hepatic integrity/function and for neutrophil infiltration. We further substantiated these results with histopathologic investigation to figure out relevant hepatic subcellular changes. On the other hand, the possibility of pharmacologic synergy with VPA was explored in a pentylenetetrazole (PTZ) mouse convulsion model. Lastly, to verify any role for DHA via kinetic interaction (clearance of VPA), we measured plasma concentrations of VPA in the presence and absence of DHA.

\section{Materials}

\subsection{Drugs and Chemicals}

Sodium valproate, a white pure powder, was a gift from Sanofi-synthelabo, Cairo, Egypt, and was dissolved in distilled water. DHA was purchased from Healthspan Co., UK, as capsules; each provides $100 \mathrm{mg}$ of pure DHA. DHA was diluted, as needed, in corn oil, with equivalent amounts of oil given to all animals and the control group. All other chemicals used were of analytical grade, and were obtained from Sigma Aldrich Chemical Co., St. Louis, MO, USA. Kits for reduced GSH, malondialdehyde and $\gamma$-glutamyl transferase $(\gamma$-GT) were obtained from BioDiagnostic, Cairo, Egypt. Kits for alkaline phosphatase (ALP), alanine aminotransferase (ALT) and albumin were obtained from ABC-Diagnostics, Cairo, Egypt. A myeloperoxidase kit was purchased from Northwest Co. (Canada) and a TNF $\alpha$ kit was from DRG Co. (USA). VPA assay ELISA kit was obtained from Dade Behring, Atterbury, Milton Keynes, UK.

\subsubsection{Animals Studies}

Adult male Sprague-Dawley rats weighing 200-250 g were used in liver toxicity study experiments. Male albino mice weighing 20-25 g were used for PTZ-epilepsy model experiments. All animals were maintained under standard conditions of temperature $\left(30^{\circ} \mathrm{C}\right)$, with a regular 12-hour light/12-hour dark cycle, and allowed free access to standard laboratory food and water. The dose used for DHA, as well as time courses used in this study were in the same range and scope as those of other studies that utilized the same models. This strategy was further confirmed after appropriate preliminary experiments. All animal care and experimental procedures were approved by the Animal Ethics Committee of Mansoura University, Mansoura, Egypt (MUEC-8-91), which is in accordance with the Principles of Laboratory Animals Care (NIH publication No. 85-23, revised 1985). 


\subsection{Rat Liver Toxicity Studies}

\subsubsection{Experimental Design}

Different animal groups, of 6-8 rats each, received the antiepileptic drug (VPA), with and without the DHA, daily for a total period of 2 weeks. Rat groupings and protocols were conducted as detailed:

\begin{tabular}{ll}
\hline Control & Received vehicle for the same period of time \\
VPA & Received VPA alone $(500 \mathrm{mg} / \mathrm{kg}$ orally [PO], daily) \\
VPA + DHA & $\begin{array}{c}\text { VPA }(500 \mathrm{mg} / \mathrm{kg} \text { PO, daily), then after } 1 \text { hour } \\
\text { received DHA }(250 \mathrm{mg} / \mathrm{kg} \mathrm{PO})\end{array}$ \\
\hline
\end{tabular}

Animals were anesthetized and blood samples were collected after 1 and 2 weeks of treatment via the orbital sinus. Serum was separated by centrifugation at 2,000 rpm for 10 minutes at $4{ }^{\circ} \mathrm{C}$. All liver markers (in serum) were measured after 1 and 2 weeks of VPA treatment; except for albumin which was monitored only after 2 weeks in virtue of its known long half-life $\left(T_{1 / 2}\right)$ value that hinders imminent short-term changes in its serum levels. Parameters measured in liver tissue were taken only after the second week of treatment (when animals were killed). Thus, liver was quickly removed and washed in an ice-cold isotonic saline, dissected, weighed, and minced. A $10 \%(\mathrm{w} / \mathrm{v})$ homogenate was made in phosphate-buffered saline (PBS) ( $\mathrm{pH}$ 7.4) for the assay of GSH and liver lipid peroxide (MDA). A consistent piece from each liver was collected in a formalin solution for histopathologic evaluations.

\subsection{Biochemical Determinations}

All enzymes, oxidative stress and hepatic synthesis markers were determined spectrophotometrically using appropriate kits. Protocols used were according to the recommended manufacturer procedures.

\subsubsection{Mouse Acute, Pentylenetetrazole (PTZ), Anticonvulsant Studies}

Mouse groups, of eight animals each, were randomly constituted. Four such groups received DHA orally, 1 hour before PTZ $85 \mathrm{mg} / \mathrm{kg}$ was injected subcutaneously (SC). The positive control group received the $\mathrm{ED}_{50}$ (dose effective in $50 \%$ of tested mice) of VPA $(175 \mathrm{mg} / \mathrm{kg}, \mathrm{PO})$, as determined by preliminary experiments. PTZ was injected 30 minutes after VPA administration, a time proven to allow peak plasma VPA level to be reached. The combination group received the DHA then VPA doses, respectively, at 30-minute intervals before $\mathrm{PTZ}$ was given (see next scheme).

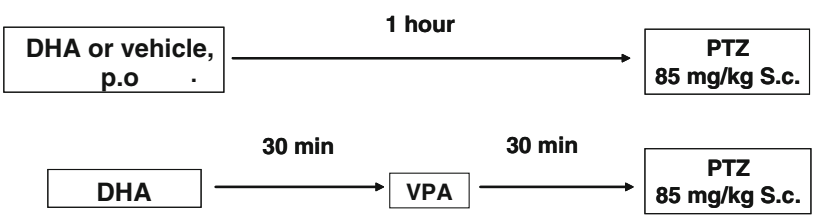

Details for mouse groupings and their drug treatments are tabulated here:

\begin{tabular}{|c|c|}
\hline Negative control & $\begin{array}{l}\text { Received equivalent amount of vehicle } \\
\text { (corn oil, PO) } 1 \text { hour before PTZ } \\
\text { ( } 85 \mathrm{mg} / \mathrm{kg} \mathrm{SC} \text { ) was injected }\end{array}$ \\
\hline VPA & $\begin{array}{l}\text { Received VPA (175 mg/kg PO) } 30 \text { minutes } \\
\text { before PTZ ( } 85 \mathrm{mg} / \mathrm{kg} \mathrm{SC} \text { ) was injected }\end{array}$ \\
\hline DHA1 & $\begin{array}{l}\text { Received DHA }(120 \mathrm{mg} / \mathrm{kg} \mathrm{PO}) 1 \text { hour } \\
\text { before PTZ ( } 85 \mathrm{mg} / \mathrm{kg} \mathrm{SC}) \text { was injected }\end{array}$ \\
\hline DHA2 & $\begin{array}{l}\text { Received DHA ( } 200 \mathrm{mg} / \mathrm{kg} \mathrm{PO}) 1 \text { hour } \\
\text { before PTZ ( } 85 \mathrm{mg} / \mathrm{kg} \mathrm{SC}) \text { was injected }\end{array}$ \\
\hline DHA3 & $\begin{array}{l}\text { Received DHA }(250 \mathrm{mg} / \mathrm{kg} \mathrm{PO}) 1 \text { hour } \\
\text { before PTZ }(85 \mathrm{mg} / \mathrm{kg} \mathrm{SC}) \text { was injected }\end{array}$ \\
\hline $\mathrm{VPA}+\mathrm{DHA}$ & $\begin{array}{l}\text { Received DHA ( } 250 \mathrm{mg} / \mathrm{kg} \text { PO), VPA } \\
(175 \mathrm{mg} / \mathrm{kg} \text {, after } 30 \text { minutes }) \text {, then PTZ } \\
\text { was injected after another } 30 \text { minutes }\end{array}$ \\
\hline
\end{tabular}

\section{Time Course and Kinetic Parameters for Serum VPA Levels in Rats, in Presence and Absence of DHA}

Rats received VPA $(200 \mathrm{mg} / \mathrm{kg})$ alone or in combination with DHA $(250 \mathrm{mg} / \mathrm{kg})$. DHA was given 1 hour before VPA. Blood samples were collected (from orbital sinus) at 30 minutes, 1 hour, 3 hours, and 6 hours after VPA was given. Samples were centrifuged and the separated serum was used for determination of VPA concentrations by enzyme immunoassay, as detailed next.

\subsection{Rat Grouping and Treatment Protocols for Pharmacokinetic Studies}

Quantitative analysis of VPA was based on a homogeneous enzyme-immunoassay technique that measures both free and protein-bound VPA in serum. The assay is fully automated through a programmed protocol that 


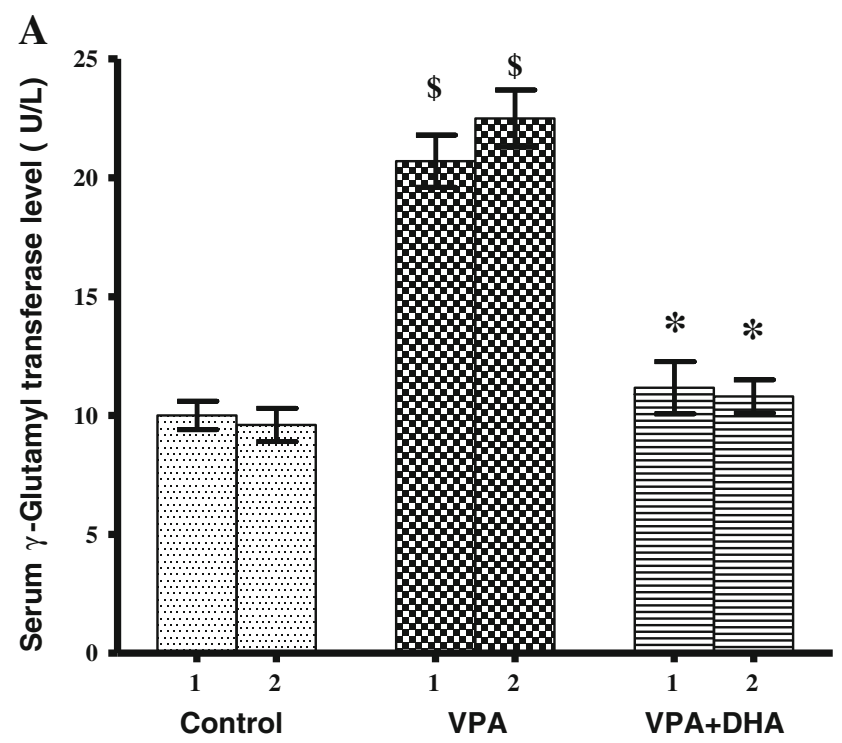

B

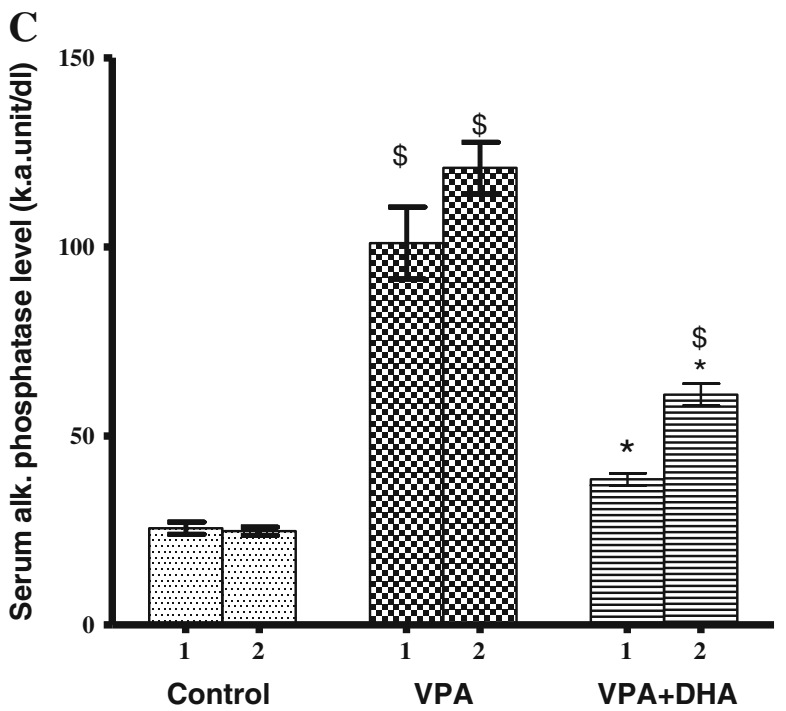

D

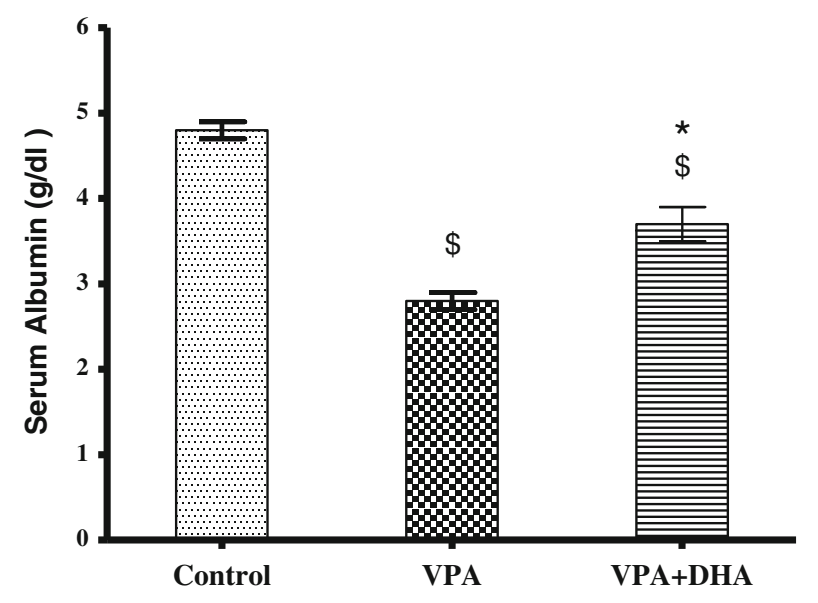

Fig. 1 a-d Effect of VPA (500 mg/kg daily/2 weeks) with and without DHA (250 mg/kg/day) on serum hepatic enzyme and albumin levels. DHA was given orally $1 \mathrm{~h}$ after VPA, then blood was withdrawn from the orbital sinus for determination of enzymes (a-c; $\gamma$-GT, ALT, ALP, respectively) after 1 and 2 weeks, or albumin (d),

utilizes a Dad Behring instrument. The results are calculated automatically by the analyzer, based on a standard curve that is constructed concurrently with the assay of samples.

\section{Statistical Analyses}

Distribution of the data was verified to be normal using Tests of Normality (SPSS package). Statistical significance was tested by one-way analysis of variance (ANOVA)

after 2 weeks. Data represent the mean \pm SEM of each group; $n=6-8$. Symbols indicate significance against VPA-treated group (asterisks) and normal control group (dollar symbols), $\gamma-G T \gamma-$ glutamyl transferase, $A L T$ alanine aminotransferase, $A L P$ alkaline phosphatase, $D H A$ docosahexaenoic acid, $V P A$ valproate

followed by Bonferroni post hoc analysis. Statistical significance was predefined at $p<0.05$.

\section{Results}

Treatment with valproate $(500 \mathrm{mg} / \mathrm{kg}$, daily $)$ for 1-2 weeks disrupted liver cell integrity as reflected by marked (2- to 5-fold) rises in serum ALT, $\gamma$-GT, and ALP (Fig. 1a-c). Such enzyme levels did not significantly vary when VPA treatment was extended from 1 to 2 weeks. Likewise, as measured by serum albumin level; the 


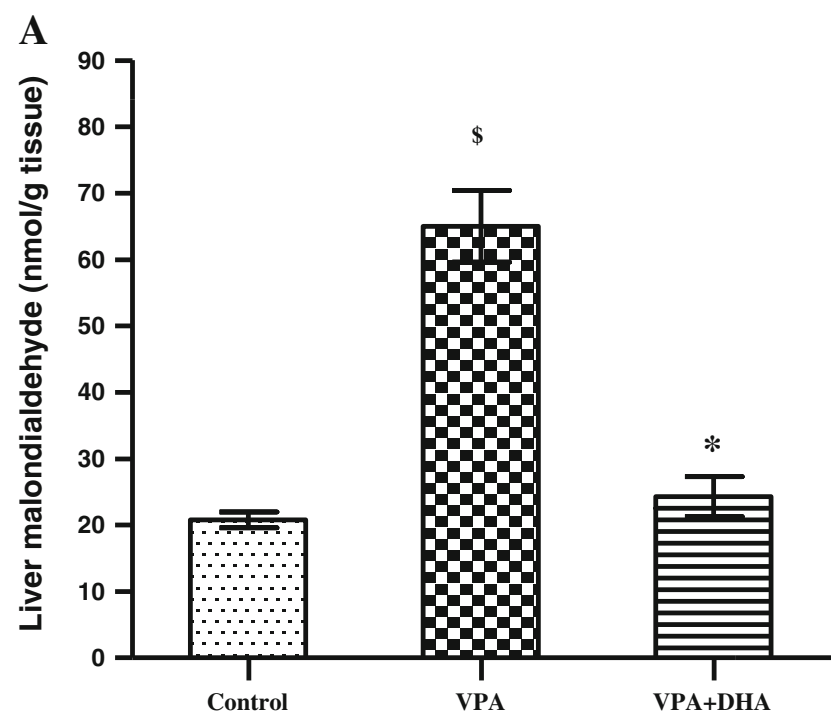

Fig. 2 a, b Effect of VPA (500 mg/kg daily/2 weeks) with and without DHA ( $250 \mathrm{mg} / \mathrm{kg} /$ day) on liver lipid peroxide (MDA) (a), and reduced glutathione $(G S H)$ (b) levels. After 2 weeks of treatment, animals were sacrificed and a $10 \% \mathrm{~W} / \mathrm{V}$ liver homogenate was

hepatocellular synthetic capacity was notably reduced (by $40 \%$ ) (Fig. 1d).

To gain insights into the hepatic molecular and cellular changes occurring following VPA treatment; oxidative stress and endogenous antioxidant levels were monitored, and histopathologic examination of the liver was also conducted. Figure 2a demonstrates that VPA evoked a 3 -fold rise in MDA levels. This was also accompanied by $35 \%$ reduction in levels of endogenous cellular protector: reduced GSH, Fig. 2b.

Downstream from hepatocellular disruption and oxidative stress, we also investigated whether VPA liver intoxication had involved inflammatory signals and/or neutrophil infiltration into the liver; and if so, how these signals may be modified by DHA. Accordingly, in liver cell homogenates, VPA upregulated the expression of proinflammatory cytokine TNF $\alpha$ (5-fold, $p<0.05$ ). This was paralleled by a $\sim 6.1$-fold rise in this cytokine level in the serum $(p<0.05$, Fig. 3a, b). Considering time-course dependency, DHA managed to blunt the rise in $\mathrm{TNF} \alpha$ effectively, after both 1 and 2 weeks, although effects of DHA were more pronounced after 1 week. Co-treatment with DHA largely suppressed the VPA-induced hepatocytic production of $\mathrm{TNF} \alpha$ in both the liver and the serum, implying also that rises in the serum are most likely linked to those in the liver. Moreover, an enzyme marker of neutrophil infiltration with known contributions to both inflammation and oxidative stress, that is myeloperoxidase (MPO), had an appreciably enhanced activity in liver homogenates $(4.2$-fold; $p<0.05)$. This response was

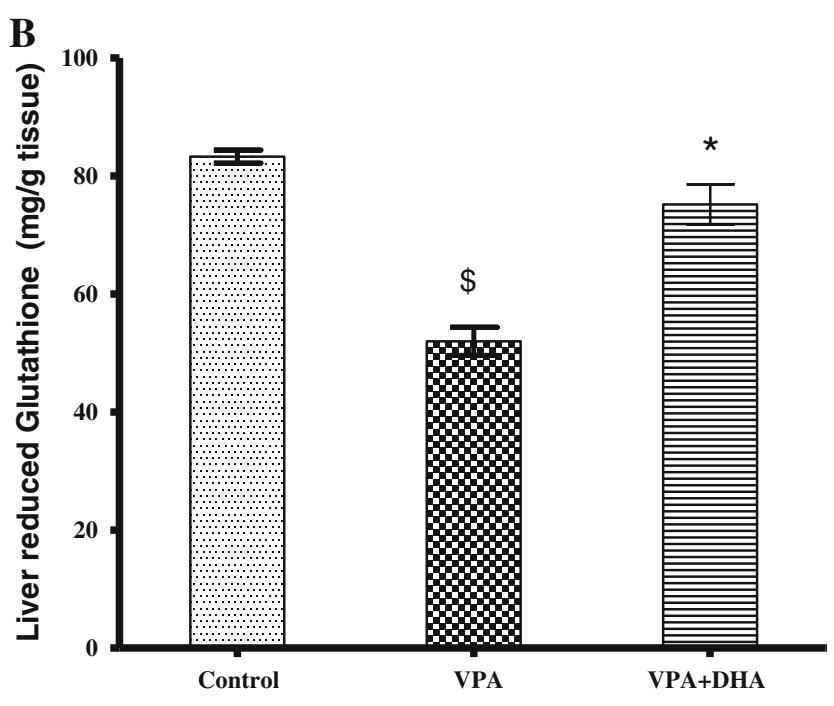

assayed for its content of MDA or GSH. Data represent the mean \pm SEM of each group; $n=7$. Symbols indicate significance against VPA-treated group (asterisks) and normal control group (dollar symbols), DHA docosahexaenoic acid, VPA valproate

likewise highly sensitive to co-treatment with DHA $(p<0.05)$, thus also revealing the versatility whereby DHA protects liver cells against VPA-induced injury.

Figure 4 represents necropsies of the liver to assess the pathological changes in the studied animals. The negative control group showed average size and color of the liver with no detected histopathologic abnormalities (photos 1, 2). Conversely, the VPA-treated group showed grossly enlarged pale livers with significantly increased weights over control values. Besides, multiple foci of focal lytic necrosis were detected in which replacement by both inflammatory cells and cellular degeneration had occurred (photo 3). Moreover, combined macrovesicular and microvesicular steatosis were evident in the periportal zone of four animals (out of six) of this group (photo 4). Concurrent treatment with DHA significantly alleviated the hepatic cellular and molecular anomalies entailed by VPA treatment. This was manifested as reduced serum liver enzymes (better after 1 than 2 weeks), lipid peroxide generation, and increased levels of hepatic GSH and serum albumin, consonant with promoted liver defensive mechanisms and enhanced protein synthesis. Furthermore, when combined with VPA, DHA showed only minimal small focal necrosis/apoptosis (single cell death) with no evidence of degeneration or steatosis (photo 5); consistent with amelioration of pathologic anomalies by DHA.

Because DHA recently demonstrated some neuroinhibitory effects on its own [18], it was of current interest to also seek possible synergy with anticonvulsant effects of VPA. Figure 5 shows that DHA elicited a dose-responsive 

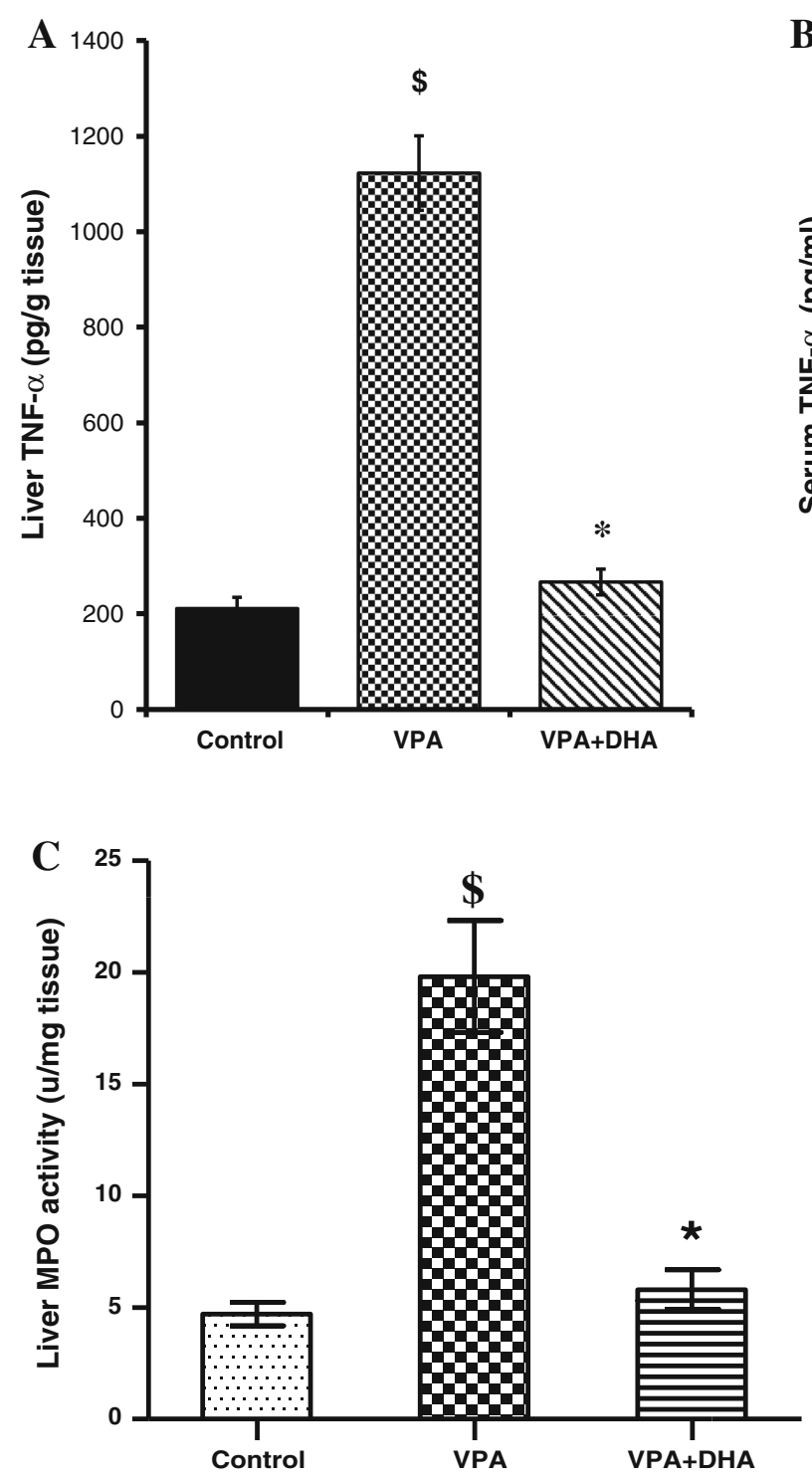

Fig. 3 Effect of VPA (500 mg/kg daily/2 weeks) with and without DHA $(250 \mathrm{mg} / \mathrm{kg} /$ day $)$ on liver and serum levels of TNFa (a, b), and on liver activity of myeloperoxidase $(M P O)$ (c). Blood was withdrawn for determination of TNF $\alpha$ after 1 and 2 weeks of treatments. Animals were sacrificed after 2 weeks and a $10 \% \mathrm{~W} / \mathrm{V}$

increase in latency (onset) of mouse tonic convulsions, with significance from control value elicited at $(250 \mathrm{mg} / \mathrm{kg}$, $p<0.05$ ), a response that was also comparable to that evoked by VPA at its $\mathrm{ED}_{50}$ dose (13.8 vs $\left.14.9 \mathrm{~min}\right)$. Combining the two FAs at such lower doses triggered a notable synergy in the latency of convulsion (32.8 min, $p<0.05)$. Similar effects for DHA were observed for the myoclonic aspect of PTZ convulsion (data not shown).

It was next worthwhile investigating whether the protective and synergistic effects of DHA involve pharmacokinetic interaction with VPA, that is, alteration of VPA clearance rate. To this end, plasma VPA levels were

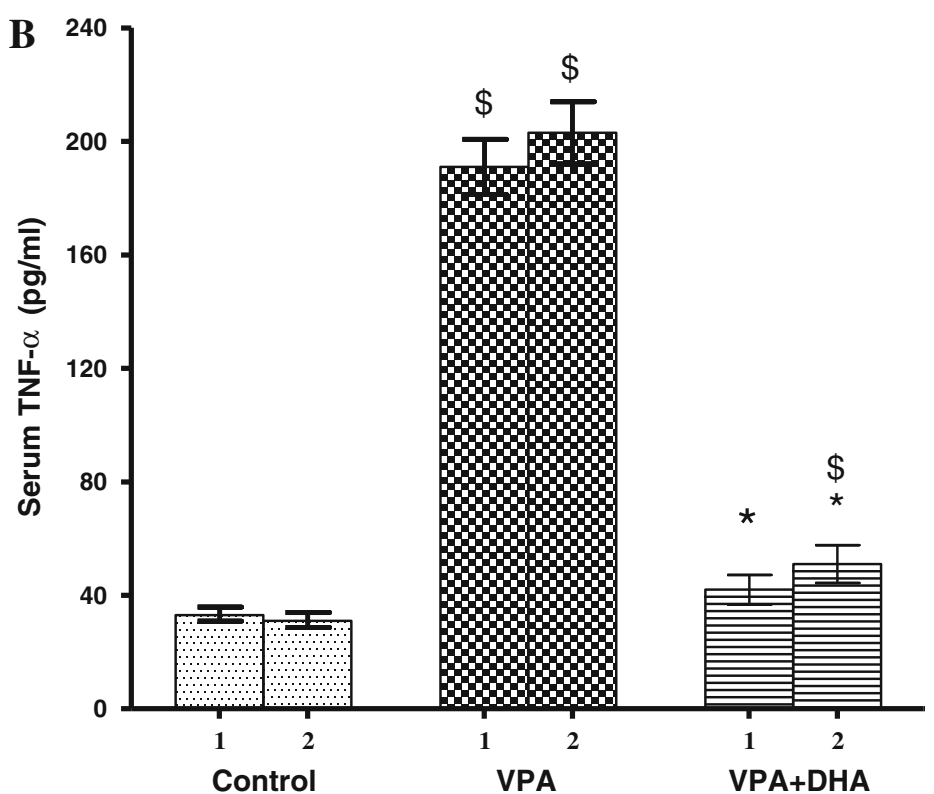

liver homogenate was assayed for both parameters. Data represent the mean \pm SEM of each group; $n=8$. Symbols indicate significance against VPA-treated group (asterisks) and normal control group (dollar symbols), DHA docosahexaenoic acid, TNF $\alpha$ tumor necrosis factor alpha, VPA valproate

determined over a time frame of 6 hours in both the presence and absence of DHA $(250 \mathrm{mg} / \mathrm{kg})$, a dose that was proven protective in earlier toxicological studies. Various kinetic parameters such as area under the curve (AUC) and volume of distribution $\left(V_{\mathrm{d}}\right)$ are displayed in Table 1 . As judged by statistical analyses, neither the peak/trough values nor the magnitude of other measured points was altered in animals given a combination of DHA and VPA, as compared with those given VPA alone. These findings unequivocally exclude the possibility of pharmacokinetic interaction and, instead, indicate peculiar dynamic effects for DHA. 
Fig. 4 Necropsies of the liver of studied animals from each group to assess the pathologic changes. Photos 1, 2 are for the negative control group, showing average size/color of the liver with no detected histopathologic abnormalities. Photo 3: VPA control group showing grossly enlarged pale livers with multiple foci of focal lytic necrosis with replacement by inflammatory cells and hepatocyte degeneration. Also, combined macrovesicular and microvesicular steatosis occurring in the periportal zone were evident in four animals in this group (photo 4). DHA when combined with VPA showed only minimal small focal necrosis with no evidence of degeneration (photo 5). DHA docosahexaenoic acid, $V P A$ valproate

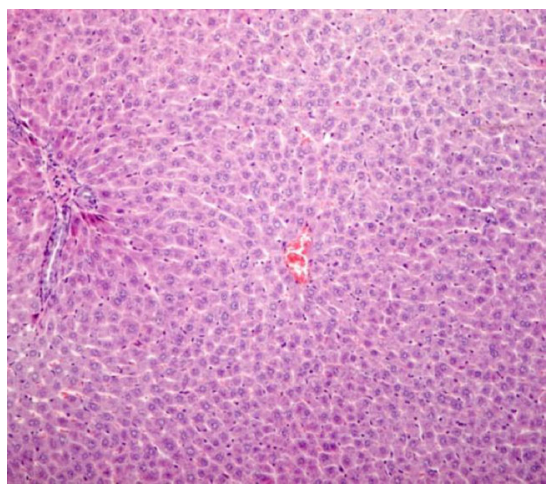

Photo. (1): Normal liver: There is central vein and the portal tract to the left. (H\&E X200)

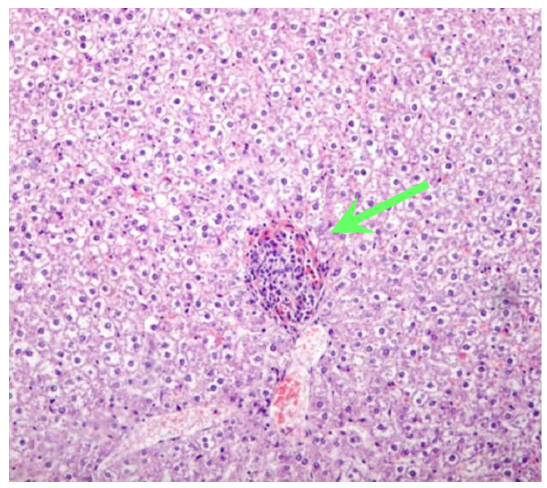

Photo. (3): VPA-control group. Focal pericentral necrosis with replacement by inflammatory cells (arrow). The surrounding hepatocytes show degenerative changes

(H\&E, X100).

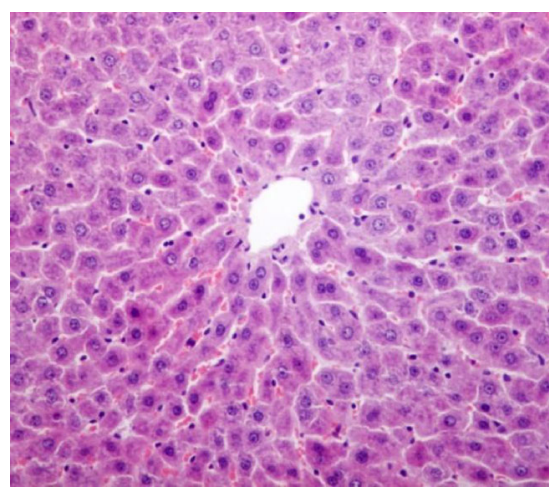

Photo. (2): Normal liver: Higher magnification with central vein surrounded by cords of normal hepatocytes. (H\&E X200)

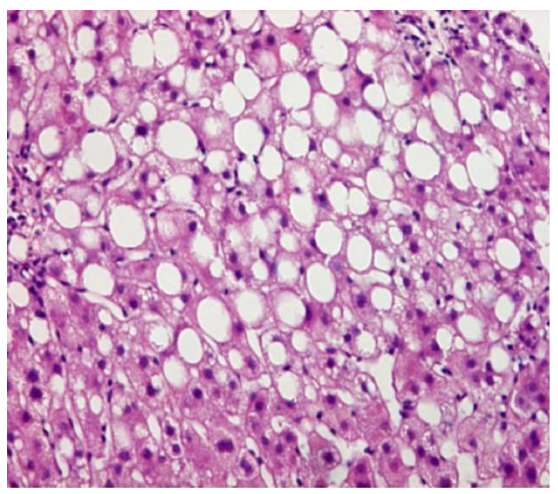

Photo. (4): VPA-control group. It shows macrovesicular and microvesicular steatosis. (H\&E, X200).

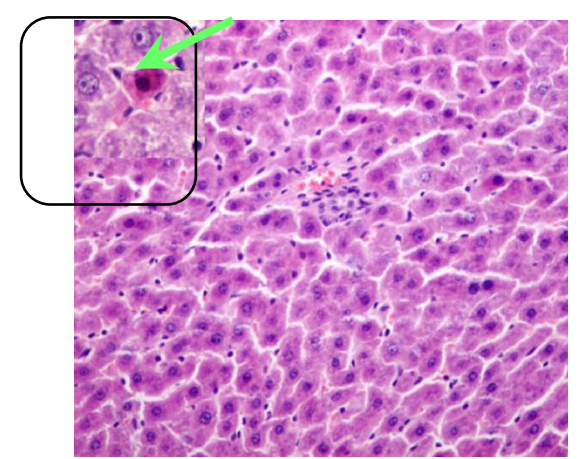

Photo. (5): VPA group treated with DHA. Minimal focal pericentral necrosis. In sight is high power magnification showing apoptosis (arrow).

(H\&E, X200, X400).

\section{Discussion}

This study reports a prominent protection by DHA against VPA-induced hepatic dysfunction, cellular anomalies, necrosis and steatosis. Likewise, it reveals that DHA enhances the anticonvulsant effects of VPA in a PTZ animal-convulsion model. These favorable effects for DHA do not target the kinetic profiles or distribution pattern of VPA, but rather trigger specific dynamic mechanisms.

Because the liver is the main drug/xenobiotic metabolic engine of the body, it is very much vulnerable to drug toxicity $[21,22]$. In particular, antiepileptic drugs (AED) have many such serious untoward reactions, as seen with VPA, phenytoin, and carbamazepine. Though relatively 


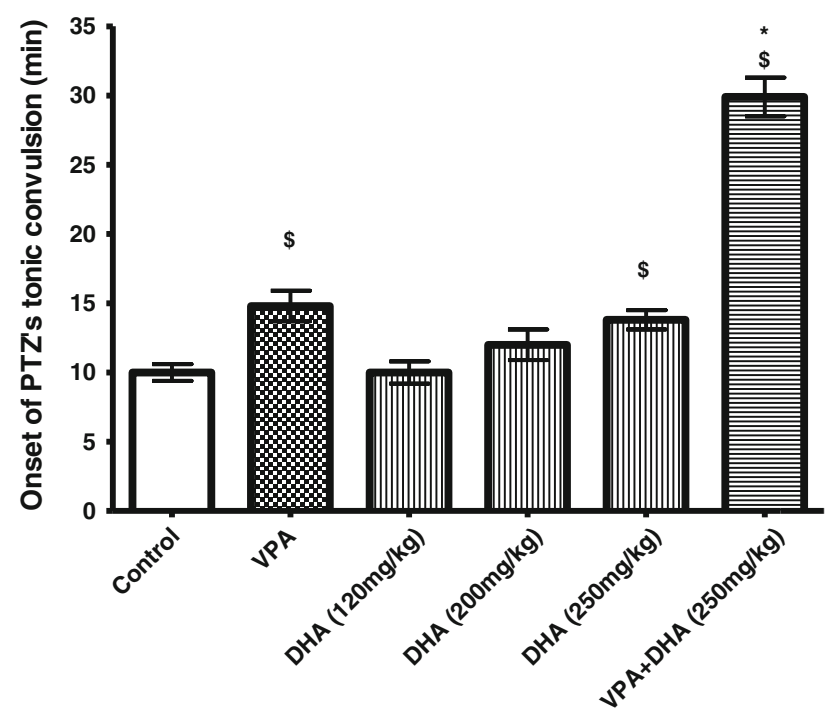

Fig. 5 Individual and combined effects of VPA $(175 \mathrm{mg} / \mathrm{kg})$ and DHA (100-250 mg/kg) on onset of tonic convulsion (min) evoked by PTZ $(85 \mathrm{mg} / \mathrm{kg})$. PTZ was injected $30 \mathrm{~min}$ after VPA administration. The combination groups received DHA then VPA, respectively; at 30 min intervals, before PTZ was given. Data represent mean \pm SEM of times recorded for each group (8 animals). Symbols indicate significance against VPA-treated group (asterisks) and normal control group (dollar symbols), DHA docosahexaenoic acid, PTZ pentylenetetrazole, $V P A$ valproate

rare, when compared with other consistently known hepatotoxic drugs, the consequences encountered with AED can cause death or an acute liver failure that would require liver transplantation. The molecular underpinnings of these anomalies to AED pertain either to production of reactive toxic metabolite/s or to induction of paradoxical immunoallergic reactions [23].

Aspects of hepatotoxicity associated with VPA have been fully unfolded [10]. Type I VPA-mediated hepatic injury is associated with a dose-dependent rise in serum liver enzymes and decline in plasma albumin. Type II VPA-mediated hepatotoxicity is a fatal, irreversible idiosyncratic reaction that is characterized by microvesicular steatosis and necrosis [11]. Although the mechanisms involved are not fully characterized, a large body of evidence suggests that reactive VPA metabolites (i.e., 4-eneVPA and its subsequent metabolite, 2,4-diene-VPA) may mediate the hepatotoxicity by inhibiting mitochondrial $\beta$-oxidation of FAs. Further, excessive generation of reactive oxygen species (ROS) (such as peroxides and hydroxyl radical) may follow the toxicity of VPA as a consequence of disrupting the liver antioxidant machinery $[10,24,25]$.

Although DHA has demonstrated protection against some drug-induced systemic toxicity [17], its impact on VPA-induced liver injury has never been sought. These views prompted us to evaluate whether, and how, DHA may obliterate VPA hepatotoxicity. Accordingly, when DHA was jointly given with VPA, serum liver marker enzyme levels (ALP, ALT and $\gamma$-GT) significantly declined, thereby suggesting the utility of DHA in protecting liver cell integrity and maintaining healthy biliary outflow. Further, DHA raised serum albumin levels, consonant with restoration of liver protein synthetic capacity. More such clues were provided from the present histopathologic studies, which depicted the capacity of DHA to ameliorate VPA-evoked hepatocellular degeneration, infiltration of inflammatory cells, induction of focal pericentral necrosis, and micro/macrovesicular steatosis.

Next, it was both worthy and intriguing to unravel the cellular and molecular means whereby DHA abates VPAevoked liver injury. Thus, DHA markedly replenished hepatic GSH levels to near baseline and blunted lipid peroxide (MDA) levels, thereby alleviating VPA-induced oxidative stress. In support, in animal models of alcohol fatty liver, DHA terminated oxidative stress and mitochondrial dysfunction [25]. Besides, human nutritional studies in prevention of heart diseases revealed that supplementation with a daily $200-800 \mathrm{mg}$ DHA enhanced its incorporation into LDL, thereby reducing its susceptibility to oxidation and accumulation of lipid peroxides [26, 27].

The possible second molecular trigger for hepatic protection by DHA is an anti-inflammatory and lipotropic effect. Inflammation and hepatic accumulation of triglycerides can foster/exacerbate oxidative stress and liver cell damage. DHA reportedly gets incorporated into liver cells, and can evidently suppress hepatic gene expression of proinflammatory cytokines $[16,20,28]$. Because hepatic necrosis, apoptosis, and steatosis were recently linked with $\mathrm{TNF} \alpha$ and its TNFR1 receptor in response to B-virus hepatitis [29, 30], it was of present interest to assess levels of this cytokine in VPA-intoxicated animals. Additionally,

Table 1 Computed pharmacokinetic parameters following administration of VPA $(200 \mathrm{mg} / \mathrm{kg}$, PO) alone or in combination with DHA $(250 \mathrm{mg} / \mathrm{kg} \mathrm{PO})$ in rats

\begin{tabular}{lllllll}
\hline Group & AUC $(m g . \mathrm{h} / \mathrm{L})$ & $C_{\max }(\mathrm{mg} / \mathrm{L})$ & $T_{\max }(\mathrm{h})$ & $T_{1 / 2}(\mathrm{~h})$ & $V_{\mathrm{d}} / F(\mathrm{~L} / \mathrm{kg})$ & $\mathrm{Cl} / F(\mathrm{~L} / \mathrm{h} / \mathrm{kg})$ \\
\hline VPA & $404.3 \pm 22.1$ & $107.6 \pm 6.6$ & 0.5 & $2.11 \pm 0.1$ & $1.518 \pm 0.11$ & $0.505 \pm 0.03$ \\
VPA + DHA & $409.6 \pm 12.8$ & $110.1 \pm 3.2$ & 0.5 & $2.04 \pm 0.12$ & $1.436 \pm 0.07$ & $0.491 \pm 0.02$ \\
\hline
\end{tabular}

$A U C$ area under serum concentration-time curve, $C_{\max }$ maximum plasma concentration, $C l$ clearance, $D H A$ docosahexaenoic acid, $F$ oral availability, $P O$ orally, $T_{1 / 2}$ elimination half-life, $T_{\max }$ time needed to attain $C_{\max }, V_{d}$ apparent volume of distribution, $V P A$ valproate 
$\omega$-3 FAs can specifically activate the peroxisome proliferator-activated receptor- $\alpha$ (PPAR $\alpha$ ), a transcriptional activator of FA oxidation in peroxisomes and mitochondria [31]. Thus, current evaluations of TNF $\alpha$ were further substantiated by the reported interaction between TNF $\alpha$ and PPAR $\alpha$ [32]. In this vein, TNF $\alpha$ was implicated in downregulating PPAR $\alpha$, thereby inducing hepatic steatosis [33]. We detected several-fold rises in hepatic TNF $\alpha$ levels following VPA treatment, a response that was appreciably blocked with DHA, implying that this $\omega-3$ FA also protects the liver via a specific anti-inflammatory mechanism. Because we also showed here the capacity of DHA (a PPAR $\alpha$ agonist) to suppress expression of TNF $\alpha$ and reduce hepatic inflammation/steatosis, these findings further establish a concept of 'cross-talk' between the TNF $\alpha$ and PPAR $\alpha$ systems in VPA-intoxicated liver cells. Further, DHA blunted the activity of a neutrophil-specific proinflammatory/pro-oxidant enzyme (MPO). Together, these findings demonstrate new effector players that are recruited by VPA to induce hepatic injury, while also attest to the diversity of the molecular basis whereby DHA can reverse these insults to ultimately elicit liver protection.

An additional objective in this study was to evaluate the possibilities of DHA synergy with anticonvulsant effects of VPA, so as to infer whether lower doses of VPA (certainly less toxic) can be therapeutically applied. Thus far, clinically, DHA is recognized to be essential for normal growth and development, and has demonstrated therapeutic benefits against some central disease states/models [16]. More recently, in a rat model, DHA was shown to raise the threshold of convulsion, suggesting its utility in the management of epilepsy. Likewise, supplementation with $\omega-3$ FAs was efficacious in the amelioration of depressive symptoms in elderly patients $[18,19]$.

Therefore, we first demonstrated that DHA evoked doseresponsive anticonvulsant effects against PTZ-induced seizures when given alone at $250 \mathrm{mg} / \mathrm{kg}$. Furthermore, when co-administered with VPA, the latency in onset of convulsion was greater than their individual responses, thereby revealing a superb synergic response. Thus, these current findings suggest the use of less hepatotoxic concentrations of VPA, while preserving its pharmacologic efficacy. At the molecular level, though neuroinhibitory targets for DHA are still incompletely defined, evidence suggests that $\omega-3$ FAs can cause inhibition of sodium and calcium voltage-gated ion channels. Additionally, the production of anti-inflammatory metabolites, like neuroprotectin-D1, has also been suggested to reduce neuroinflammation, thereby raising the seizure threshold and abating convulsions in response to $\omega-3$ FAs $[34,35]$.

Collectively, the above-mentioned effects for DHA clearly depict the involvement of cellular 'dynamic' effectors in protection against VPA-induced liver injury.
However, currently these findings cannot exclude the involvement of metabolic/kinetic means whereby DHA may modulate plasma levels/clearance of VPA. This view is also supported by earlier findings that both DHA and VPA can individually evoke kinetic interactions with many other drugs, thereby altering their efficacies [35-38]. Hence, it was indeed both challenging and intriguing to probe these possibilities for the present combination regimen (DHA/VPA). We found that co-treatment with DHA had no effect on serum VPA concentration at different time intervals, as compared with animals that had received VPA only. Likewise, no significant statistical difference was observed in the VPA pharmacokinetic parameters generated in the presence and absence of DHA, thus unequivocally indicating that DHA had no effect on clearance rate of VPA. Although the hepatoprotective effects of DHA were observed with another drug, paracetamol [39], this study not only revealed some molecular underpinnings and synergy effects for DHA actions, but also ruled out any sort of kinetic interactions with VPA, an important drug efficacy aspect.

Conclusively, DHA is an ideal aide in synergy with VPA that acts via dynamic mechanisms to abate VPA-induced hepatic injury, while also largely enhancing its anticonvulsant effects, thus potentially allowing lower doses of VPA to be applied. Notably also, the known kinetic profiles and safety reports on DHA largely support these findings.

Accordingly, it becomes evident that a rational design/ exploitation of synergy via the use of phytomedicals should enrich modern pharmacotherapy enough to revolutionize the management of vicious adverse drug reactions, as typically exemplified here by VPA-evoked hepatic injury [40]. Clinically, data from this study suggest a fruitful drug regimen to reduce hepatic injury. This is governed by the capacity of DHA to restore normal liver function and integrity, and to synergize with neuroinhibitory (antiepileptic) effects to enable lower doses of VPA. Together, this combined drug regimen should augment the overall therapeutic index of VPA.

Acknowledgments This study was supported in part by a postgraduate fellowship award to (M.A.E ${ }^{1}$ ) from Mansoura University, Egypt; and by an American Heart Association SDG grant to (A.A.E-M ${ }^{2}$ ).

Open Access This article is distributed under the terms of the Creative Commons Attribution Noncommercial License which permits any noncommercial use, distribution, and reproduction in any medium, provided the original author(s) and the source are credited.

\section{References}

1. El-Mowafy AM, Al-Gayyar MM, El-Mesery ME, Salem HA, Darweish MM. Novel chemotherapeutic and renal protective effects for 
the green-tea (EGCG): role of oxidative stress and inflammatorycytokine signaling. Phytomedicine. 2010;17:1067-75.

2. Calder PC. n-3 Fatty acids, inflammation, and immunity relevance to postsurgical and critically ill patients. Lipids. 2004;39(12):1147-61.

3. El-Mowafy AM, Alkhalaf M. Resveratrol activates adenylyl-cyclase in human breast-cancer cells: a novel, estrogen receptor-independent cytostatic mechanism. Carcinogenesis. 2003;24(5):869-73.

4. Einarson TR. Drug-related hospital admissions. Ann Pharmacother. 1993;27(7-8):832-40.

5. Johannessen CU, Johannessen SI. Valproate: past, present, and future. CNS Drug Rev. 2003;9(2):199-216.

6. Bedry R, Parrot F. Severe valproate poisoning. Réanimation. 2004;13:324-333.

7. Zimmerman RI, Ishak KG. Valproate-induced hepatic injury. Analyses of 23 fatal cases. Hepatology. 1982;2:591-7.

8. Cotarlu D, Zaldman JL. Valproic acid and the liver. Clin Chem. 1988;34(5):890-7.

9. Graf WD, Oleinik OE, Glauser T. Altered antioxidant enzyme activities in children with a serious adverse experience related to valproic acid therapy. Neuropediatrics. 1998;29:195-201.

10. Tong V, Thomas KH, Frank S. Valproic acid. Time course of lipid peroxidation biomarkers, liver toxicity, and valproic acid metabolite levels in rats. Toxicol Sci. 2005;86(2):427-35.

11. Spiller HA, Krenzelok EP, Klein-Schwartz W, Winter ML, Webe JA, Sollee DR, et al. Multicenter case series of valproic acid ingestion: serum concentrations and toxicity. J Toxicol Clin Toxicol. 2000;38(7):755-60.

12. Tang W, Borel AG, Fujimiya T, Abbott FS. Fluorinated analogues as mechanistic probes in valproic acid hepatotoxicity: Hepatic microvesicular steatosis and glutathione status. Chem Res Toxicol. 1995;8(5):671-82.

13. Raza M, Al-Bekairi AM, Ageel AM, Qureshi S. Biochemical basis of sodium valproate hepatotoxicity and renal tubular disorder. Pharmacol Res. 1997;35(2):153-7.

14. Buchi KN, Gray PD, Rollins DE, Tolman KG. Protection against sodium valproate injury in isolated hepatocytes by alphatocopherol and $N, N^{\prime}$-diphenyl- $p$-phenylenediamine. J Clin Pharmacol. 1984;24(4):148-54.

15. Lheureux PE, Hantson P. Carnitine in the treatment of valproic acid-induced toxicity. Clin Toxicol (Phila). 2009;47(2):101-11.

16. Simopoulos AP. Essential fatty acids in health and chronic diseases. Forum Nutr. 2003;56:67-70.

17. El-Mesery ME, Al-Gayyar MM, Salem HA, Darweish MM, ElMowafy AM. Chemopreventive and renal protective effects for docosahexaenoic acid (DHA): implications of CRP and lipid peroxides. Cell Div. 2009;4(1):6.

18. Taha AY, Jeffrey MA, Taha NMY, Bala S, Burnham WM. Acute administration of docosahexaenoic acid increases resistance to pentylenetetrazole-induced seizures in rats. Epilepsy Behav. 2010;17:336-43.

19. Rondanelli M, Giacosa A, Opizzi A, Pelucchi C, La Vecchia C, Montorfano G, Negroni M, Berra B, Politi P, Rizzo AM. Effect of omega-3 fatty acids supplementation on depressive symptoms and on health-related quality of life in the treatment of elderly women with depression: a double-blind, placebo-controlled, randomized clinical trial. J Am Coll Nutr. 2010;29(1):55-64.

20. Ishii H, Horie Y, Ohshima S, Anezaki Y, Kinoshita N, Dohmen $\mathrm{T}$, et al. Eicosapentaenoic acid ameliorates steatohepatitis and hepatocellular carcinoma in hepatocyte-specific Pten-deficient mice. J Hepatol. 2009;50(3):562-71.

21. Bissell DM, Gores GJ, Laskin DL, Hoofnagle JH. Drug-induced liver injury: mechanisms and test systems. Hepatology. 2001;33:1009-13.

22. Pessayre D, Berson A, Fromenty B. Mitochondria in steatohepatitis. Semin Liver Dis. 2001;21:57-69.
23. Björnsson E. Hepatotoxicity associated with antiepileptic drugs. Acta Neurol Scand. 2008;118(5):281-90.

24. Szalowska E, van der Burg B, Man HY, Hendriksen PJ, Peijnenburg AA. Model steatogenic compounds (amiodarone, valproic acid, and tetracycline) alter lipid metabolism by different mechanisms in mouse liver slices. PLoS One. 2014;9(1):e86795. doi:10.1371/journal.pone.0086795.

25. Higgins S, Carroll YL, McCarthy SN. Susceptibility of LDL to oxidative modification in healthy volunteers supplemented with low doses of $\mathrm{n}-3$ polyunsaturated fatty acids. $\mathrm{Br} \mathrm{J}$ Nutr. 2001;85:23-31.

26. Song BJ, Moon KH, Olsson NU, Salem N Jr. Prevention of alcoholic fatty liver and mitochondrial dysfunction in the rat by longchain polyunsaturated fatty acids. J Hepatol. 2008;49(2):262-73.

27. Calzada C, Colas R, Guillot N, Guichardant M, Laville M, Véricel E, et al. Subgram daily supplementation with docosahexaenoic acid protects low-density lipoproteins from oxidation in healthy men. Atherosclerosis. 2009;208(2):467-72.

28. Schmocker C, Weylandt KH, Kahlke L, Wang J, Lobeck H, Tiegs G. Omega-3 fatty acids alleviate chemically induced acute hepatitis by suppression of cytokines. Hepatology. 2007;45:864-9.

29. Kim JY, Song EH, Lee HJ, Oh YK, Choi KH, Yu DY, Park SI, Seong JK, Kim WH. HBx-induced hepatic steatosis and apoptosis are regulated by TNFR1- and NF-kappaB-dependent pathways. J Mol Biol. 2010;397(4):917-31.

30. Ding WX, Yin XM. Dissection of the multiple mechanisms of TNF-alpha-induced apoptosis in liver injury. Cell Mol Med. 2004;8(4):445-54.

31. Cullingford TE, Dolphin CT, Sato H. The peroxisome proliferator-activated receptor alpha-selective activator ciprofibrate upregulates expression of genes encoding fatty acid oxidation and ketogenesis enzymes in rat brain. Neuropharmacology. 2002;42(5):724-30.

32. Beier K, Völkl A, Fahimi HD. TNF-alpha downregulates the peroxisome proliferator activated receptor-alpha and the mRNAs encoding peroxisomal proteins in rat liver. FEBS Lett. 1997;412(2):385-7.

33. Vreugdenhil M, Bruehl C, Voskuyl RA, Kang JX, Leaf A, Wadman WJ. Polyunsaturated fatty acids modulate sodium and calcium currents in CA1 neurons. Proc Natl Acad Sci USA. 1996;93(22):12559-63.

34. Bazan NG. Omega-3 fatty acids, pro-inflammatory signaling and neuroprotection. Curr Opin Clin Nutr Metab Care. 2007;10(2):136-41.

35. Hirunpanich V, Sato H. Docosahexaenoic acid (DHA) inhibits saquinavir metabolism in-vitro and enhances its bioavailability in rats. J Pharm Pharmacol. 2006;58(5):651-8.

36. Hirunpanich V, Katagi J, Sethabouppha B, Sato H. Demonstration of docosahexaenoic acid as a bioavailability enhancer for CYP3A substrates: in vitro and in vivo evidence using cyclosporin in rats. Drug Metab Dispos. 2006;34(2):305-10.

37. Phua LC, New LS, Goh CW, Neo AH, Browne ER, Chan EC. Investigation of the drug-drug interaction between alpha-lipoic acid and valproate via mitochondrial beta-oxidation. Pharm Res. 2008;25(11):2639-49.

38. Chung JY, Cho JY, Yu KS, Kim JR, Lim KS, Sohn DR, et al. Pharmacokinetic and pharmacodynamic interaction of lorazepam and valproic acid in relation to UGT2B7 genetic polymorphism in healthy subjects. Clin Pharmacol Ther. 2008;83(4):595-600.

39. Meganathan M, Madhana MG, Sasikala P, Mohan J, Gowdhaman $\mathrm{N}$, Balamurugan $\mathrm{K}$, et al. Evaluation of antioxidant effect of Omega 3-fatty acid against paracetamol-induced liver injury in albino rats. Global J Pharmacol. 2011;5(1):50-3.

40. Wagner H, Ulrich-Merzenich G. Synergy research: approaching a new generation of phytopharmaceuticals. Phytomedicine. 2009;16(2-3):97-110. 